\title{
APLICAÇÃO DO MÉTODO DE ELEMENTOS FINITOS PARA ANÁLISE DO DESLOCAMENTO ESTRUTURAL ESTÁTICO DE UMA FRESADORA CNC
}

MARANGONI, Antônio Carlos ${ }^{1}$

BOMFIM, Camila Colombari

MARANGONI, Rafael Junqueira ${ }^{3}$

RESUMO: Verifica-se uma demanda cada vez maior por máquinas que substituam o trabalho manual. Naturalmente, percebe-se que elas não somente devem executar 0 trabalho, mas, também, ter atributos de precisão cada vez maior no desempenho. No presente trabalho, temos o projeto de uma fresadora equipada com comando numérico computadorizado (CNC), equipamento que será estudado a partir da rigidez dos eixos utilizados para movimentos lineares. Tais eixos serão examinados a fim de conhecer a deflexão estática existente. A nossa hipótese é a de que esta deflexão é causada pelo próprio peso das partes móveis existentes na máquina, mobilidade muito utilizada para esse tipo de observação em geometria complexa. O método empregado foi o de elementos finitos, em conjunto com computadores para a simulação, comparando-se o resultado com critérios existentes em literatura, estabelecendo a precisão de máquinaferramenta. Como resultados parciais, vimos que a deflexão causa imperfeições no paralelismo da peça usinada e, verificando-se tal problema, propomos que os valores mensurados das imperfeições geométricas podem ser inseridos ao CNC da máquina, obtendo, assim, melhor precisão na forma exigida da peça.

PALAVRAS-CHAVE: Fresadora, deflexão, análise de elementos finitos

ABSTRACT: There is an increasing demand for machines that replace manual labor. Naturally, it is clear that they must not only perform the job, but also have attributes of increasing precision in performance. In the present work, we have the design of a milling machine equipped with a computer numerical command $(\mathrm{CNC})$, equipment that will be studied from the rigidity of the axes used for linear movements. Such axes will be examined in order to discover the existing static deflection. Our hypothesis is that this deflection is caused by the weight of the moving parts on the machine, a mobility widely used for this type of observation in complex geometry. The method used was that of finite elements, together with computers for the simulation, comparing the result with existing criteria in the literature, establishing the machine tool precision. As partial results, we saw that the deflection causes imperfections in the parallelism of the machined part and, in view of this problem, we propose that the measured values of the geometric imperfections can be inserted into the machine's CNC, thus obtaining better precision in the required shape of the part.

KEYWORDS: Milling, deflection, finite element analysis

\footnotetext{
${ }^{1}$ Doutor em Ciências. Mestre em Bioengenharia pela EESC-USP. Possui Capacitação em EAD pela UNIFEI. Graduado em: Física; Química, Ciências Físicas Biológicas, Matemática e Pedagogia Plena. Docente da UEMG, Unidade Frutal - MG, nos cursos de Engenharia Agronômica, Tec. Alimentos e Administração. Contato: antonio.marangoni@uemg.br.

${ }^{2}$ Bacharel em Engenharia Mecânica pela UNIFRAN. Mestranda em Ciências. Docente da Faculdade Anhanguera, Franca - SP. Contato: colombari.camila@gmail.com.

3 Graduando em Engenharia Civil pela EESP-USP, ênfase em cálculo estrutural. Contato: rafaeljmarangoni@usp.br.
} 


\section{INTRODUÇÃO}

Uma fresadora é considerada uma máquina-ferramenta, e tem como objetivo dar diversas conformações à matéria. Ao mesmo tempo, a máquinaferramenta é muito utilizada na fabricação de inúmeras peças de diversos materiais (metálicos, plásticos e de madeira), por meio da movimentação mecânica de um conjunto de eixos, utilizando, para tal fim, uma ferramenta de corte.

Ao longo de sua história, o homem tem procurado desenvolver ferramentas para facilitar qualquer que tenha sido a sua atividade rotineira. Ao largo disso, a expansão do mercado no mundo, fez com que fossei necessário um aumento da fabricação de produtos importantes para a humanidade. E, a partir de tais demandas, é que ferramentas obsoletas passam por processos de melhoramento, coincidindo com o que Giannotti (2007) afirma, dizendo que, apesar desse contexto de evolução contínua, foi somente no século XVIII é que apareceram as primeiras máquinas do princípio da tecnologia industrial.

Outro fato importante foi o aperfeiçoamento da fabricação do ferro, sendo submetido a modificações em sua formulação e estrutura, especialmente nos séculos XVIII e XIX. E, depois, com o advento de novos materiais, advém um aumento na demanda de produtos e um consequente incremento no sistema de produção nas indústrias, dando origem às máquinas-operatrizes (NAVARRO, 2006).

No começo do século XX, aparecem estudos com objetivo de estabelecer padrões e critérios para a fabricação, avaliação e validação de máquinas-ferramenta. Leta (et al., 2005), nos dizem que toda máquinaferramenta passa por testes de avaliação e aceitação após a sua construção, momento em que são verificados vários parâmetros, com o objetivo de ter, cada vez mais, geometrias extremamente precisas ou, na pior das hipóteses, conhecer os erros geométricos que se manifestam em uma máquinaferramenta.

Com o emprego do computador na operação de uma máquinaferramenta, já no meio do século XX, aumentou ainda mais a necessidade de 
se conhecer as variações estruturais que uma máquina-ferramenta pudesse ter em sua geometria, pois, a partir do conhecimento de algum desvio estrutural, tem-se a possibilidade de inserir esses erros de geometria no sistema de controle numérico computadorizado (CNC), repassando tais erros para o sistema de coordenadas mecânicas da máquina, fazendo com que seja corrigido tal desvio geométrico, obtendo-se, assim, precisões maiores das peças confeccionadas.

Ainda no meio do século $X X$, percebeu-se a inviabilidade do uso de sistemas numéricos, tais como o método de elementos finitos (MEF). Tal método, normalmente se empregava para se fazer a análise de estruturas complexas. O estudo destas estruturas requisitava apenas métodos analíticos, que fossem capazes de encontrar deformações e conhecer a rigidez das estruturas, inclusive, fracionando tais estruturas facilitando a análise. Atualmente, com o avanço tecnológico e com a criação de potentes sistemas de hardware e softwares, viabilizou-se o uso do método numérico. Um dos mais conhecidos é o método de elementos finitos para a utilização nessa nova tecnologia, e que, segundo Nunes (2012), torna possível que se faça a análise da rigidez estática de uma máquina-ferramenta em pouco tempo, desde que se utilize tal método ainda na fase de projeto, diminuindo, inclusive, os custos na fase inicial de projeto.

\section{MÉTODOS COMPUTACIONAIS DE ANÁLISE DE ELEMENTOS FINITOS}

Segundo Lotti (2006), o método dos elementos finitos (MEF) teve suas origens no final do século XVIII, quando Gauss propôs a utilização de funções de aproximação para a solução de problemas matemáticos.

Já, para Budynas (2011), em meados de 1940, surgiram as primeiras necessidades de cálculos mais complexos, que envolviam a mecânica estrutural com o trabalho de Hrennikoff, McHenry e Newmark, os quais usaram um reticulado de elementos lineares (barras e vigas) para a solução de tensões em sólidos contínuos. 
Entretanto, não se tem notícia da existência de alguém a quem se possa atribuir tal metodologia. Assim, engenheiros, físicos e matemáticos estiveram na órbita do desenvolvimento e da evolução, desde o início.

De acordo com Bortholin (2014), o método (MEF) foi utilizado na engenharia, em 1960, por Clough, em um problema de elasticidade plana. Originalmente o método foi implementado no estudo de tensões em aeronaves. Após os estudos de Clough, no início dos anos 60, o método dos elementos finitos começou a ser amplamente utilizado em várias áreas da engenharia, particularmente na resolução de inúmeros problemas em que já estava sendo reconhecido eficaz.

O autor ainda nos mostra que o MEF é um método de solução aproximada de equações diferenciais, em que o material analisado é discretizado (subdivido) em partes menores, ainda que mantenha as mesmas propriedades do material original. Por meio dessas equações, os problemas são descritos e resolvidos pelo modelo matemático, procurando-se obter resultados desejáveis. Assim, o objetivo é o de calcular as deformações e tensões nas partes menores, individualmente, minimizando o consumo de tempo, espaço de memória no computador e erros, oferecendo, assim, uma solução numérica para um problema físico.

Amarante (2003) acrescenta que o MEF se caracteriza como uma simulação, na qual pode ser recriado matematicamente o comportamento de um determinado sistema físico. Este método permite que o componente em estudo tenha forma geométrica, propriedades mecânicas e condições de contorno quaisquer, ocorrendo uma semelhança entre o modelo do MEF e a situação física real.

Meireles (2007) nos mostra que os métodos numéricos avançados, dos quais o método de elementos finitos (MEF) é o mais conhecido, são extremamente importantes para a definição e análise de estruturas complexas de engenharia, tais como naves espaciais, aviões, automóveis, edifícios, pontes, represas, recipientes de retenção, máquina-ferramenta etc. A modelação por elementos finitos apresenta-se hoje como um instrumento indispensável para a elaboração de projetos de engenharia. 
Souza (2003) nos mostra que a ideia principal do método dos elementos finitos consiste em se dividir o domínio (meio contínuo) do problema em sub-regiões (meio discreto) de geometria simples (formato triangular, quadrilateral, cúbico etc.), conforme ilustra esquematicamente a Figura 01. Esta ideia é bastante utilizada na engenharia, área em que usualmente tenta-se resolver um problema complexo, subdividindo sua geometria em uma série de problemas mais simples ou menores, chamados de elementos finitos e, por intermédio deste procedimento, se faz a aproximação de uma solução exata, obtida por interpolação de uma solução aproximada.

Figura 01 - Malha de elementos finitos para um problema plano

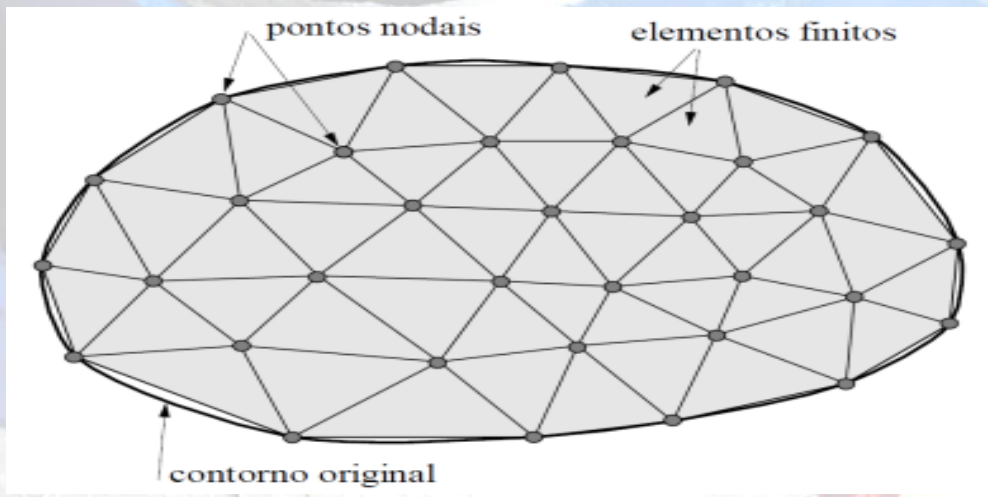

Fonte: Souza (2003)

Meireles (2007) nos mostra que na definição da forma da malha, utiliza-se a funcionalidade do programa de elementos finitos de malhagem livre. Apenas se exige que cada linha do modelo seja dividida num número igual de partes, de tal forma a que a distribuição da malha seja mais detalhada nas zonas da peça com mais pormenores geométricos. Este critério permite manter uma maior uniformidade no processo de distribuição da malha durante o cálculo para os vários modelos.

De acordo com Sánchez (2001), os problemas resolvidos pelo MEF podem ser classificados em:

- Problemas de equilíbrio - análise estática;

- Problemas de autovalor - análise dinâmica; e,

- Problemas de propagação - análise transiente. 
Apesar de brevemente, não podemos deixar de tratar de um assunto muito importante, como os três modos essenciais para a definição das etapas do MEF: pré-processamento, processamento e pós-processamento (BATHE, 1996, apud BORTHOLIN, 2014).

\section{Pré-processamento}

$\mathrm{Na}$ definição do problema e do domínio, se desenvolve o objeto e verifica o problema a ser tratado. Levam-se em consideração as condições iniciais e de contorno, carregamentos, hipóteses simplificadoras do objeto, o tipo de elemento a ser analisado, a geração de malha formada por esses elementos e a especificação das propriedades dos materiais.

Na discretização ou divisão do domínio em elementos, o objeto é subdividido em um número finito de elementos, conforme ilustra a Figura 02 , e que podem ser um triângulo ou um quadrilátero, voltado para problemas em duas dimensões, ou tetraedros e hexaedros, para três dimensões. Esses elementos são compostos por nós internos e externos, que podem estar localizados no interior ou nas arestas do mesmo. Como condição, assume-se que esses elementos estão interligados entre si, por meio de nós localizados no contorno dos mesmos. O processo de discretização do contínuo depende muito da experiência e habilidade do engenheiro.

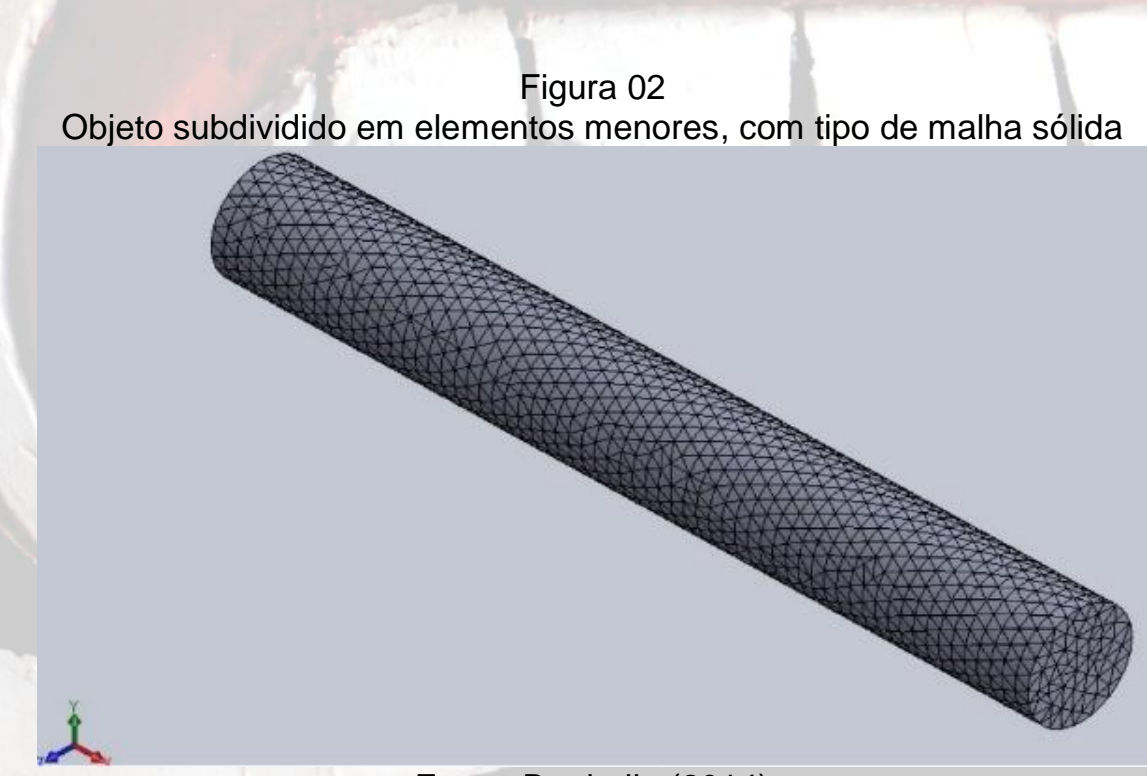

Fonte: Bortholin (2014) 


\section{Processamento}

A etapa de processamento corresponde à fase de análise propriamente dita e, de acordo com o tipo de elemento escolhido anteriormente, a entrada de dados e análise incremental é aceita. É importante determiná-las para cada problema, pois essas funções representaram a distribuição exata ou real dos deslocamentos. Normalmente a função de interpolação adotada é a polinomial, devido à facilidade na manipulação matemática.

Ainda segundo Bathe (1996, apud BORTHOLIN, 2014), é no processamento que a rigidez da estrutura é avaliada (montagem da matriz de rigidez global), baseada nos modelos matemáticos que descrevem o comportamento físico dos materiais constituintes da estrutura analisada, ou seja, os modelos constitutivos que relacionam tensão e deformação no material. A partir disto, pode-se proceder à montagem e resolução de equações algébricas que resultarão nas soluções de um campo requerido, como, por exemplo, os deslocamentos.

\section{Pós-processamento}

Nesta última etapa do método, depende-se somente das necessidades do engenheiro que está modelando o problema.

Para Bortholin (2014), as equações diferenciais resolvidas descrevem o fenômeno em estudo, podendo ser representado pelo deslocamento, deformação, gradiente de tensão de acordo com o critério de resistência adotado etc.

Essas etapas implementadas em softwares específicos permitem ao usuário investigar em qualquer parte do modelo proposto construído, os resultados provenientes da etapa anterior, de maneira simples e eficiente, facilitando a interpretação e análise dos resultados, estimando, assim, a solução do problema proposto em um curto espaço de tempo.

Os resultados de uma análise obtidos pelo MEF demonstram algumas características que fazem com que certas informações sejam fundamentais, 
por exemplo, as visualizações de campos vetoriais (deslocamentos) e de campos tensoriais (tensões e deformações).

Atualmente, os softwares apresentam também uma solução gráfica que facilita a análise do resultado. Por apresentar grandes vantagens na aplicação do método, o seu uso tem se difundido.

Podem-se citar algumas das principais vantagens disso, tais como propriedades do material em elementos adjacentes, as quais podem ser diferentes. $\mathrm{E}$, isso, é o que permite a utilização do método em conjuntos de montagens e observar as respostas diferentes nas regiões de contato.

Outra vantagem é a de que o método pode ser utilizado em geometrias complexas, não se limitando apenas a geometrias simples, sendo utilizado em modelos com contornos irregulares.

Além das já citadas, os elementos podem possuir diferentes tamanhos em regiões específicas, refinando a análise em locais críticos, minimizando a análise em pontos de baixa solicitação.

Conclui este conjunto de avanços, o fato de que o modelo permite a utilização em condições de contorno descontínuas sem grandes problemas.

\section{MATERIAIS E MÉTODOS}

A pesquisa foi desenvolvida a partir de uma fresadora construída, porém, sem projeto técnico e teórico de fabricação, utilizando como base para construção da máquina apenas informações e imagens em fóruns relacionados ao assunto.

Empregamos o software de desenho assistido por computador (CAD 3D), em três dimensões SolidWorks®, para a confecção do protótipo digital, com o objetivo de se fazer as devidas simulações estáticas de flexão na estrutura e nos eixos de movimento linear da fresadora em questão.

Os materiais utilizados têm importância no critério de flexão de uma máquina-ferramenta.

Portanto, demonstraremos no Quadro 01, as características dos materiais utilizados na estrutura da fresadora em estudo. 
Temos ilustrado, na Figura 03 abaixo, o protótipo que foi criado com o auxílio da ferramenta $C A D$, apresentando o conjunto da fresadora em estudo. Entende-se por conjunto o cabeçote, que é o conjunto de movimento linear do eixo $\boldsymbol{Z}$, o pórtico, que é o conjunto de movimento linear do eixo $\boldsymbol{X}$, e a base, que é o conjunto de movimento linear do eixo $Y$.

Figura 03 - Fresadora subdividida por conjuntos e suas massas

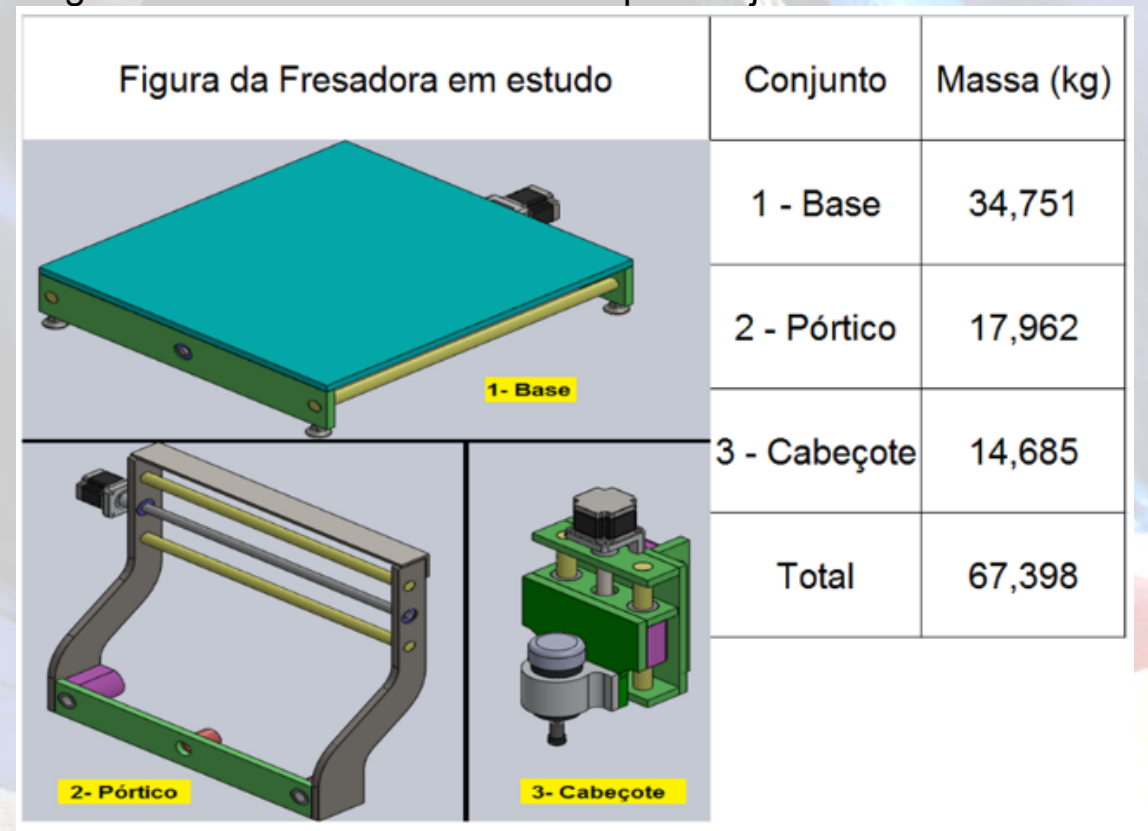

Fonte: O Autor

Há, possivelmente, duas formas de se conhecer a deflexão de uma máquina-ferramenta, ainda na fase inicial do projeto, porém, será utilizado o método numérico para se conhecer a deflexão estrutural.

Blodget (1963, apud SANT'ANNA, NUNES e GOMES, 2009) apresenta uma literatura com o critério para análise de flexão em estruturas de máquinaferramenta de precisão sendo $(\Delta / L)$, em que a resultante da viga da estrutura da máquina-ferramenta, dividido pelo comprimento da mesma viga, a deflexão permitida no centro da viga deverá estar numa faixa entre 0,00001 e $0,000001(\mathrm{~mm} / \mathrm{mm})$.

Para Shigley (2005), uma carga estática é uma força estacionária ou momento aplicado a um membro, e para ser estacionária, esta força ou momento não pode mudar em grandeza, pontos de aplicação e nem em direção de forma alguma. 
Portanto, o desenho confeccionado em CAD 3D, teve todos os seus elementos e componentes fixados no desenho exatamente no ponto central dos eixos de movimento linear, ponto esse em que, segundo Nunes (2012), quando simplificando a estrutura de uma máquina-ferramenta a um pórtico plano, que é o caso da fresadora em questão, pode se obter a deflexão máxima de um eixo ou estrutura em estudo.

Para o ensaio do presente trabalho, utilizou-se 0 software SolidWorks ${ }^{\circledR}$, com a ferramenta de elementos finitos, simulando os esforços no protótipo CAD 3D da máquina.

Aplicou-se o sistema de malhagem livre, o qual aparece com o tipo de malha fina, que pode ser visto na Figura 04, tetraédrico de ordem superior, o software automaticamente encontra o centro de massa total, aplica-se a aceleração da gravidade no conjunto atuando no valor de $9,81 \mathrm{~m} / \mathrm{s}^{2}$.

Figura 04

Protótipo coberto pelo tipo de malha utilizado na simulação

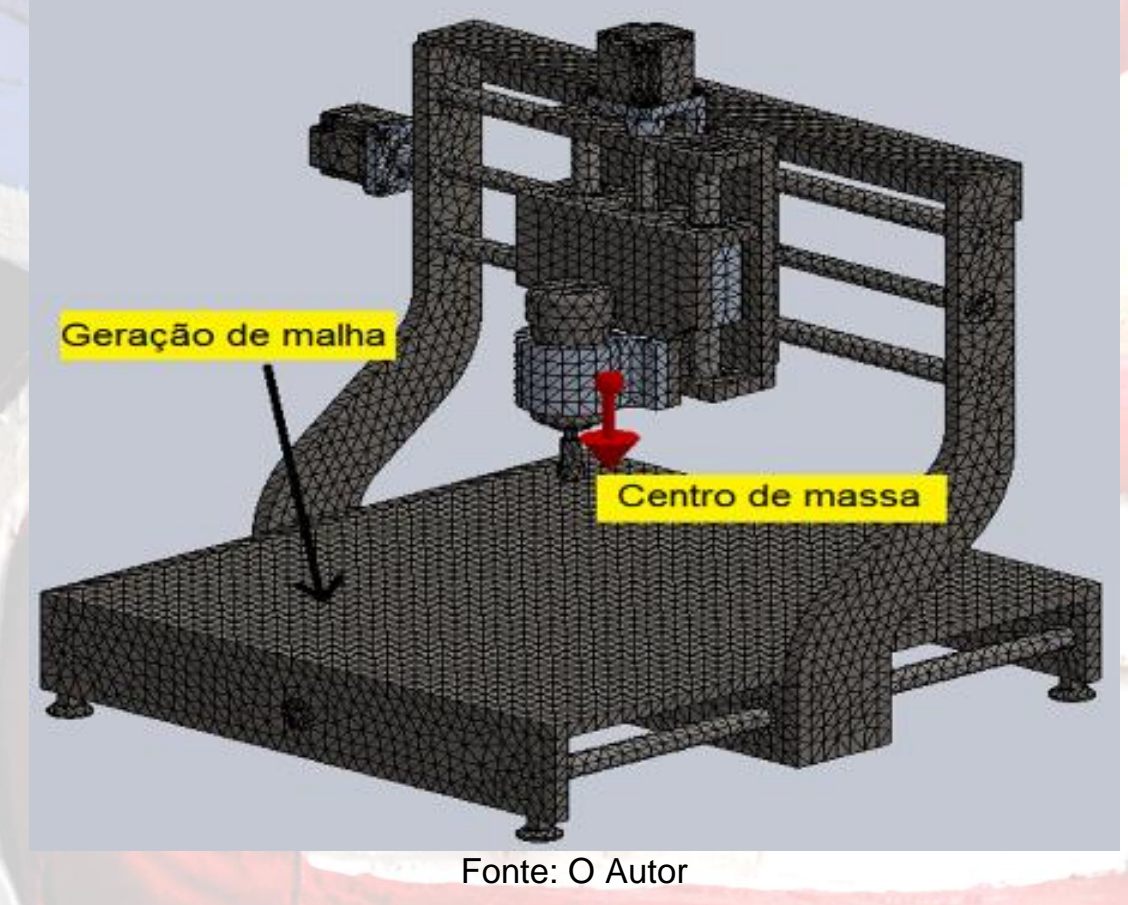

Para a análise da deflexão do eixo de movimento linear $\boldsymbol{X}$, simplificouse a estrutura da fresadora, deixando evidente somente o pórtico, que é o conjunto do eixo de movimento linear $\boldsymbol{X}$, onde aparece a deflexão a ser estudada, e o cabeçote, que é o conjunto de movimento linear do eixo $\boldsymbol{Z}$, que 
representa a carga de $144 \mathrm{~N}$, a ser aplicada para o estudo da deflexão do eixo citado.

Utilizaram-se dois pontos de fixações na parte inferior do pórtico, fixações essas que são exigidas pelo software. A Figura 05 nos ilustra esta afirmação. Para um melhor entendimento, os conjuntos pórtico e cabeçote, os eixos de movimento linear $\boldsymbol{X}$, as fixações exigidas pelo software e o sentido da carga que é aplicada.

Figura 05 - Ilustração do conjunto $\boldsymbol{X}$ em estudo

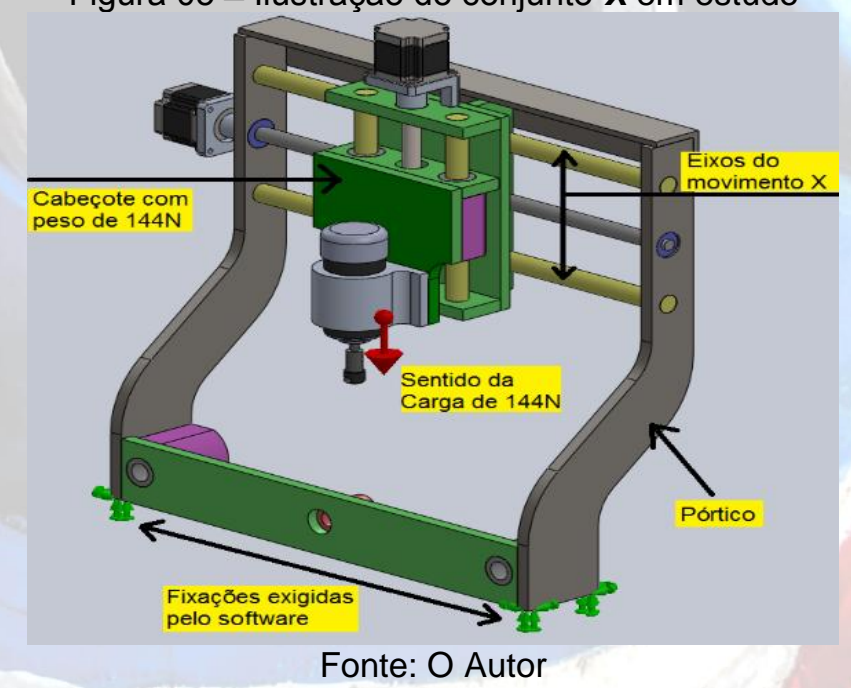

Para o estudo dos eixos de movimento linear $\boldsymbol{Y}$, tem-se a fresadora montada por inteiro, onde a base, que comporta os eixos de movimento linear $\boldsymbol{Y}$, eixos esses que estão sendo analisados nesse caso, recebe a carga simultânea dos conjuntos cabeçote com $144 \mathrm{~N}$, e pórtico com $176 \mathrm{~N}$, que representa um total de carga a ser aplicada no estudo de deflexão do eixo de movimento linear $\boldsymbol{Y}$ de $320 \mathrm{~N}$.

As fixações exigidas pelo software para esse caso estão somente nos pés de apoio da fresadora. A Figura 06 ilustra o que se atesta.

$\mathrm{E}$, para um melhor entendimento, os conjuntos pórtico e cabeçote, com suas respectivas cargas, a base com os eixos de movimento linear $\boldsymbol{Y}$, as fixações exigidas pelo software e o total e sentido da carga aplicada. 


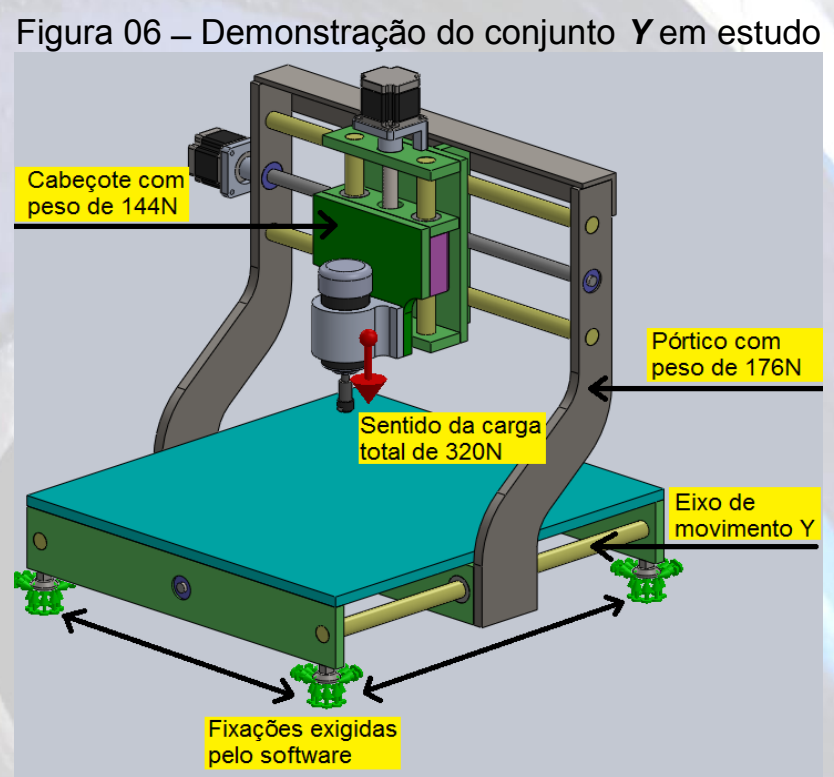

Fonte: O Autor

Por fim, temos o deslocamento total, com as características para a simulação, exemplificada na figura 06 , sendo mensurado o deslocamento total na posição do eixo-árvore, que terá como resultado, a somatória das deflexões do eixo de movimento linear $\boldsymbol{X}$ e o eixo de movimento linear $\boldsymbol{Y}$. Portanto, teremos um total de 02 simulações e 03 locais analisados, aferindo medida ao estudo do aqui descrito.

\section{RESULTADOS E DISCUSSÕES}

No caso da fresadora em estudo, a distância dos pontos de apoio dos eixos de movimentos lineares que estão na horizontal ( $\boldsymbol{X}$ e $\boldsymbol{Y}$ ) é respectivamente de $475 \mathrm{~mm}$ :

Logo: $\left(475^{*} 0,00001\right)=0,00475$ ou $4,75 \mu \mathrm{m}$

$$
\left(475^{\star} 0,000001\right)=0,000475 \text { ou } 0,475 \mu \mathrm{m}
$$

Ou seja, para enquadrar nesse critério, a deflexão em todos os locais de medida na fresadora deverá ficar entre $4,75 \mu \mathrm{m}$ a $0,475 \mu \mathrm{m}$. Para efeito de projeto, utiliza-se o maior valor, ou seja, abaixo de $4,75 \mu \mathrm{m}$, qualquer valor será aceito. 
A Figura 07, abaixo, apresenta a análise no eixo de movimento linear $\boldsymbol{X}$, juntamente com as posições dos acessórios de fixação na parte inferior do pórtico, em cor laranja, pertinente à simulação, com a aplicação da carga do cabeçote (conjunto $Z$ ) no valor de $144 \mathrm{~N}$.

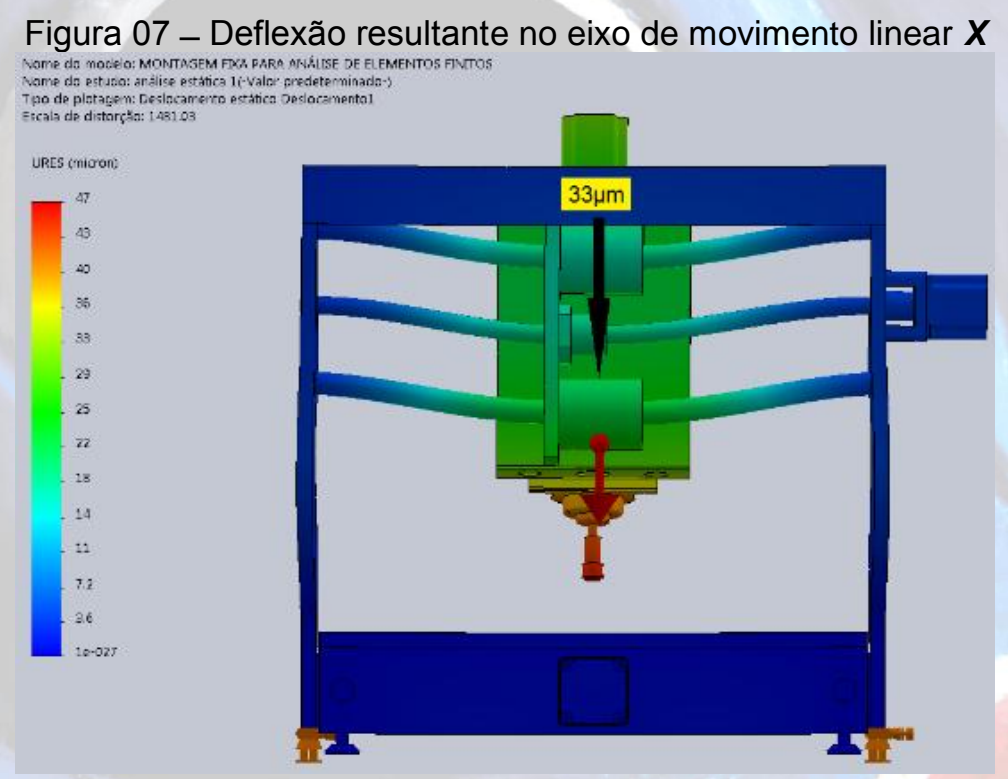

Fonte: O Autor

A Figura 08, por sua vez, apresenta a análise no eixo de movimento linear $\boldsymbol{Y}$, com os acessórios de fixação em cor verde, aplicados somente no pé de apoio da máquina, sendo a carga do pórtico e cabeçote (conjunto $\boldsymbol{X}$ e Z) aplicada no valor de $320 \mathrm{~N}$.

Figura 08 - Deflexão resultante no eixo de movimento linear $\boldsymbol{Y}$

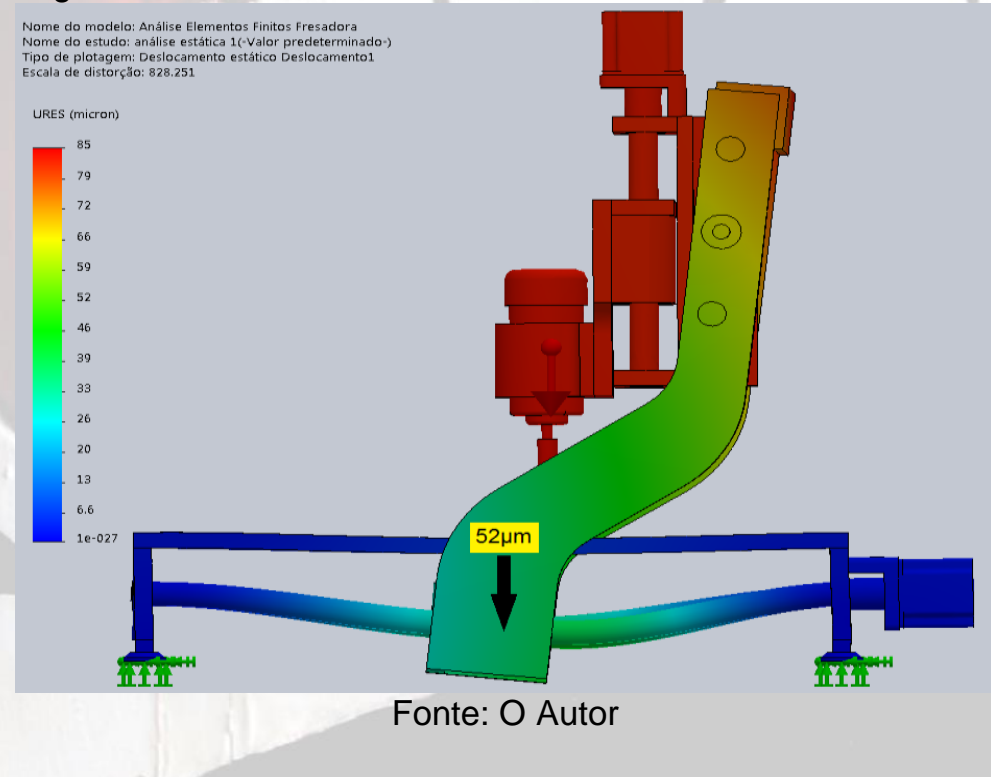


Por fim, a Figura 09, a seguir, apresenta a análise no conjunto inteiro, tendo as mesmas características de fixações e cargas da Figura 08, acima, diferenciando somente o local de medição, demonstrando o deslocamento total resultante das somatórias dos eixos, deslocamento que aparece na posição do eixo-árvore.

Figura 09 - Deflexão máxima no ponto do eixo-árvore

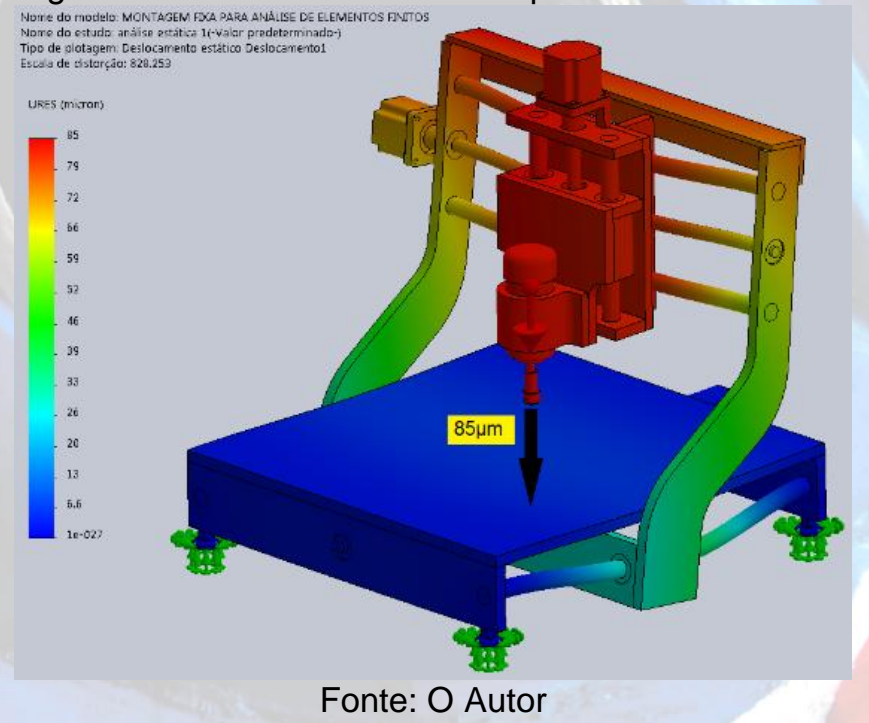

Através do critério apresentado por Blodget (1963, apud SANT'ANNA, NUNES e GOMES, 2009), a deflexão máxima permitida para o caso em estudo é de 4,75 $\mu \mathrm{m}$.

No presente estudo, obtivemos valores muito superiores em relação ao critério em todos os eixos estudados e, também, no deslocamento resultante na posição do eixo-árvore, conforme apresentou o Quadro 03.

Portanto, nota-se que a fresadora em estudo atenda a tal critério, devem ser realizadas algumas possíveis modificações na máquina, tais como: aumento do diâmetro dos eixos de movimentos lineares ou substituição dos eixos lineares por guias lineares apoiadas.

Isso poderia ser feito também por intermédio de substituições de materiais da estrutura, por materiais mais leves, ou ainda, por meio da alteração no centro de massa e um novo estudo para possível validação. 


\section{CONCLUSÃO}

Conclui-se, então, que com o valor obtido de $4,75 \mu \mathrm{m}$ para a deflexão superior, com os critérios estabelecidos. Através das simulações estáticas, chega-se a valores superiores em todas as partes mensuradas da fresadora em estudo.

Para o eixo-árvore, a somatória das deflexões dos eixos de movimentos lineares $\boldsymbol{X}$ e $\boldsymbol{Y}$ obteve um deslocamento de $85 \mu \mathrm{m}$.

Ressaltamos que as torções existentes em todo o conjunto, possivelmente foram ocasionadas pelas irregularidades do centro de massa.

Ficou evidente que a utilização de um software de simulação durante o projeto de máquina-ferramenta auxilia 0 entendimento das relações interdependentes de esforços e reduz os custos de fabricação de equipamentos fora das especificações necessárias para atender ao projeto. 


\section{REFERÊNCIAS}

AMARANTE, M. V. Análise de tensões em dentina restaurada com pinos intra-radiculares de diferentes materiais. Dissertação de Mestrado em Ciências da Engenharia Metalúrgica. Pontifícia Universidade Católica: Rio de Janeiro, 2003.

BORTHOLIN, R. C. Contribuição para aplicação de novos materiais em hastes intramedulares para o uso veterinário. Dissertação Mestrado em Engenharia Mecânica. Escola de Engenharia de São Carlos: Universidade de São Paulo, São Carlos, 2014.

BUDYNAS, R. G.; NISBETT, J.K. Elementos de Máquinas de Shigley. Projeto de Engenharia Mecânica. Porto Alegre: AMGH, 2011.

GIANNOTTI, V. História das Lutas dos Trabalhadores no Brasil. Rio de Janeiro: Mauad, 2007.

LETA, F. R. et al. Métodos de melhora de ordenação em DEA aplicados à avaliação estática de tornos mecânicos. Investigação Operacional, v.25, n.2, p. 229-242, 2005.

LOTTI, R. S. et al. Aplicabilidade científica do método dos elementos finitos. $R$ Dental Press Ortodon Ortop Facial. Maringá, v. 11, n. 2, p. 35-43, 2006. Disponível em http://www.scielo.br/revistas/dpress/paboutj.htm. Acesso em 28 de Ago. de 2014.

MEIRELES, J. F. B. Análise dinâmica de estruturas por modelos de elementos finitos identificados experimentalmente. Tese de Doutorado em Engenharia Mecânica. Universidade do Minho: Guimarães, 2007.

NAVARRO, R. F. A. Evolução dos Materiais. Parte1: da Pré-história ao Início da Era Moderna. Revista Eletrônica de Materiais e Processos. Departamento de Engenharia de Materiais da UFCG: Campina Grande, v.1, p. 01-11, 2006. Disponível em: http://www.dema.ufcg.edu.br/revista/

NUNES, J. M. Uma abordagem metodológica para seleção de máquinaferramenta para o fresamento de componentes estruturais aeronáuticos. Tese de Doutorado de Ciências em Engenharia Aeronáutica e Mecânica. Instituto Tecnológico de Aeronáutica: São José dos Campos, 2012.

SÁNCHEZ, C. A. A. Estudo de impacto usando Elementos Finitos e análise não linear. Dissertação de Mestrado em Engenharia Mecânica. Escola de Engenharia de São Carlos: Universidade de São Paulo, São Carlos, 2001.

SANDVIK@. Configuração de máquinas-ferramenta - Número de eixos. 2014. Disponível em: http://www.sandvik.coromant.com/en$\mathrm{gb} / \mathrm{knowledge/milling/getting}$

started/machines for milling/pages/default.aspx. Acesso em 04 de Set. de 2014. 
SANT'ANNA, D. R.; NUNES, J. M.; GOMES, J. O. Análise estrutural de máquinas-ferramenta pelo método dos elementos finitos, In: ENCONTRO DE INICIAÇÃO CIENTÍFICA E POS-GRADUAÇÃO DO ITA, 15, 2009. São José dos Campos. Anais do $15^{\circ}$ Encontro de Iniciação Científica e Pós-Graduação do ITA. São José dos Campos: ITA, 2009.

SCHROETER, E. R. B.; STOETERAU, R. L.; Weingaertner L. Apostila de Processos de Usinagem: fabricação por remoção de material. 2004.

SERVO, J. C. P. Seleção de um material alternativo para a estrutura de máquinas-ferramentas de arranque de apara. Dissertação de Mestrado em Engenharia Mecânica. Universidade do Minho: Guimarães, 2013.

SHYGLEY, J. E.; MISCHKE, C. R.; BUDYNAS, R. G. Projeto de engenharia mecânica. 7. ed. Porto Alegre: Bookman, 2005.

SOUZA, R. M. O Método dos Elementos Finitos Aplicado ao Problema de Condução de Calor. UFPA: Belém, 2003. 